\title{
Syntheses and Adsorption Property Research of N-Diethyl Imidazole Modified PS Resins for Iodine
}

\author{
Wanxing Luo, Xiaoyan Zhao, Moon Luo \\ Yantai Darbond Technology Co. Ltd., Yantai, China \\ Email:1wx0015@gmail.com
}

Received June 22, 2012; revised July 23, 2012; accepted August 7, 2012

\begin{abstract}
A new N-diethyl imidazole modified PS resins was synthesized in this paper. The composition, structure, and properties of the resulting resins were studied by means of Fourier transform infrared (FTIR) and elemental analysis methods. The results showed that the resins hold the adsorption function group of quaternary ammonium structure. Meanwhile, the adsorption properties of the resulting resins for iodine were investigated. The results showed that the uptake of iodine found to be higher at natural water than at acidic medium; the adsorption capacity increased with the increasing of temperature, but when the temperature is higher than $30^{\circ} \mathrm{C}$, adsorption capacity didn't increase; the adsorption kinetics of the resins can be modeled by pseudo first-order rate equation and pseudo second-order rate equation wonderfully. Both Freundlich equations and Langmuir equations could well interpret the adsorption of resins for iodine.
\end{abstract}

Keywords: N-Diethyl Imidazole; PS; Iodine; Adsorption

\section{Introduction}

Iodine, as sterilization materials, has the advantage of high efficiency, low toxicity, long acting time and no resistance to bacteria, so it is valuable in sterilization applications. Ellis [1] searched the iodine inhibition of Escherichia coli active performance, showed that add 3 - 8 $\mathrm{mg} / \mathrm{L}$ of iodine into poor water conditions can obtain drinking water. However, iodine is poor dissolution in water, which restricts the application in sterilization. In order to expand the scope of application, iodine can load on certain media materials, when it is used, it can be released gradually from media materials into water, and then killing various microorganisms. Polyvinyl alcohol, polyvinyl pyrrolidone, poly2-vinyl pyridine, poly4-vinyl pyridine [2-7] are excellent polymer adsorption materials, these polymer load with iodine can overcoming the default of poor dissolution in water, which expanded the application range of iodine. These materials such as polyvinyl alcohol, polyvinyl pyrrolidone and other materials are water soluble, which can be used only once, in order to obtain reusable adsorbent materials, a great deal of search has been conducted, In this paper we focused our interest in getting reusable iodine adsorption materials and its absorption property for iodine, these materials are water insoluble, but can release iodine to act as antibacterial materials. Normally, iodine can compared with $\mathrm{I}^{-}$ to form $\mathrm{I}_{3}^{-}, \mathrm{I}_{5}^{-}, \mathrm{I}_{7}^{-}$, which can compared with quaternary ammonium salt structures with the cationic attract mechanism to form adsorption. Quaternary ammonium salt cationic has a bactericidal action which can kill bacteria without release of reactive reagent into water $[8,9]$, so these material load with iodine has very strong bactericidal activity, the sterilization usage will be discussed in another article.

In this paper, chloromethylated polystyrene resins (CMPS) was obtained through chloromethylation of polystyrene, then N-ethyl imidazole was immobilized onto the resins to form N-diethyl imidazole modified PS resins, thereby obtaining the iodine adsorption capacity. Adsorption test showed that the result resins have good adsorption properties for iodine.

\section{Experimental}

\subsection{Reagents and Apparatus}

Macro porous PS, Cross-linking Degree 7.5\%, was purchased from Wandong Chemical Factory, China. Chlorine ether, $-\mathrm{CH}_{2} \mathrm{Cl}$ content $42 \%$, was purchased from Sangdong Hengjia Chemical Co., China. Other reagents were analytical-grade chemical products and used without further purification.

Infrared spectra were recorded on a Magana-IR 550 (series II) Fourier transform-infrared spectrometer, Nicolet Co., USA, test conditions: potassium bromide pellets, scanning 32 times, resolution is $4 \mathrm{~cm}^{-1}$.

The content of $\mathrm{Cl}$ elementary analysis was performed on an Elementar Varioel III instrument, Elementar Co., Germany. 
The absorption of iodine analysis was performed on a Shimadzu 2550 UV-vis Spectrophotometer instrument, Shimadzu Co., Japan.

\subsection{Preparation of the Resins}

The PS beads (about $2.4 \mathrm{~g}$ ) were swollen in $14.4 \mathrm{ml}$ of Chlorine ether for $12 \mathrm{~h}$. Then $1.44 \mathrm{~g}$ of $\mathrm{ZnCl}_{2}$ was added and the mixture was magnetically stirred at $38^{\circ} \mathrm{C}$ for 60 minutes under the protection of $\mathrm{N}_{2}$. Finally, the product was washed with ethanol, then it was transferred to Soxhlet's extraction apparatus for reflux-extraction in $95 \%$ ethanol for $12 \mathrm{~h}$, and then was dried under vacuum at $50^{\circ} \mathrm{C}$ over $48 \mathrm{~h}$. The obtained product was chloromethylated polystyrene resins.

The CMPS beads (about $4 \mathrm{~g}$ ) were swollen in $80 \mathrm{ml}$ of $\mathrm{N}$-ethyl imidazole for $12 \mathrm{~h}$, then the mixture was magnetically stirred at $80^{\circ} \mathrm{C}$ for $24 \mathrm{~h}$. Finally, the product was washed with ethanol. Then it was transferred to Soxhlet's extraction apparatus for reflux-extraction in 95\% ethanol for $12 \mathrm{~h}$, and then was dried under vacuum at $50^{\circ} \mathrm{C}$ over $48 \mathrm{~h}$. The product obtained was N-diethyl imidazole modified PS resins (CMPS2EZ).

The synthesis routine of the resins was shown in Scheme 1.

\subsection{Drawing Standard Curves}

All UV-vis spectra were recorded on a Shimadzu UV2550 spectrophotometer, and Standard curves were obtained with absorbance as the longitudinal coordinates and the contention of $\mathrm{I}_{3}^{-}$as the abscissa.

\subsection{Absorption Capacity}

The sorption of iodine in CMPS2EZ was studied by equilibrating $5 \mathrm{mg}$ of CMPS2EZ resins with $50 \mathrm{ml}$ of iodine solution for $45 \mathrm{~h}$ with constant stirring. The UVvis spectra of $2 \mathrm{ml}$ iodine solution taken before and after equilibration were recorded after diluted to $25 \mathrm{ml}$. The capacity of the resins was calculated.

\subsection{Optimum pH of Iodine Uptake}

For studying the iodine uptake capacity of CMPS2EZ resins in strong acidic media, $\mathrm{HCl}, \mathrm{CH}_{3} \mathrm{COOH}$ or $\mathrm{H}_{2} \mathrm{SO}_{4}$ was used, so different acid of iodine solution was pre-

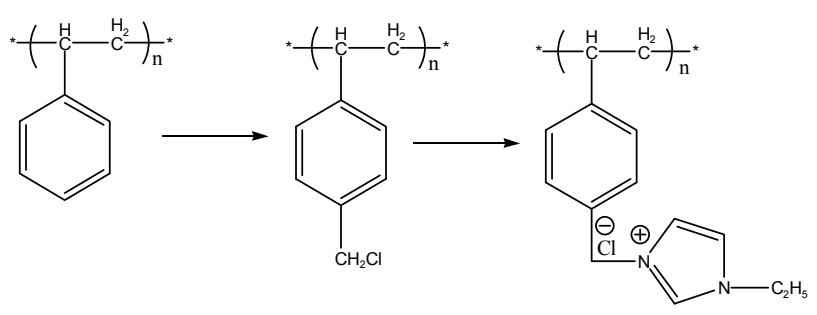

Scheme 1. Synthesis routine of CMPS2EZ. pared with different acid. The sorption of iodine in the CMPS2EZ resins was studied by equilibrating $5 \mathrm{mg}$ of CMPS2EZ resins with $50 \mathrm{ml}$ of iodine solution for $45 \mathrm{~h}$ with constant stirring. The UV-vis spectra of $2 \mathrm{ml}$ iodine solution taken before and after equilibration were recorded after diluted to $25 \mathrm{ml}$.

\subsection{Sorption Kinetics}

The rate of loading of iodine on CMPS2EZ resins was determined under the following conditions: $50 \mathrm{ml}$ of iodine solution was stirred with $5 \mathrm{mg}$ of CMPS2EZ resins at certain temperature in a mechanical shaker. An aliquot of $2 \mathrm{ml}$ iodine solution was removed at predetermined intervals for analysis by UV-vis spectra after diluted to $25 \mathrm{ml}$, iodine loaded on CMPS2EZ resins was calculated.

\subsection{Adsorption Thermodynamics}

CMPS2EZ resins were soaked in iodine solution at certain temperature, $5 \mathrm{mg}$ of CMPS2EZ resins was equilibrated with $50 \mathrm{ml}$ of iodine solution and The UV-vis spectra of $2 \mathrm{ml}$ iodine solution taken before and after equilibration were recorded after diluted to $25 \mathrm{ml}$.

\section{Results and Discussion}

\subsection{Infrared Spectra Characterization}

Figure 1 shows the Infrared Spectra of PS, CMPS and CMPS2EZ. By comparison with the curve of PS resins, the characteristic peak of CMPS resins at 676 and 1263 $\mathrm{cm}^{-1}$ appear, which is the characteristic peak of $\mathrm{C}-\mathrm{Cl}$ bond and $\mathrm{C}-\mathrm{H}$ bond in $-\mathrm{CH}_{2} \mathrm{Cl}$, indicating that $-\mathrm{CH}_{2} \mathrm{Cl}$ groups have been introduced into the PS resins successfully. By comparison with the curve of CMPS resins, the characteristic peak of $\mathrm{C}-\mathrm{Cl}$ at $676 \mathrm{~cm}^{-1}$ almost disappears in the curve of CMPS2EZ, and two new broad peaks appears at $1654 \mathrm{~cm}^{-1}$ and $1087 \mathrm{~cm}^{-1}$, which is the characteristic peak of $\mathrm{N}$-diethyl imidazole bond, indicating that $\mathrm{N}$-diethyl imidazole groups have been introduced into the CMPS2EZ resins successfully.

\subsection{Elemental Analysis of CMPS Resins}

Elemental analysis results show that the chlorine content

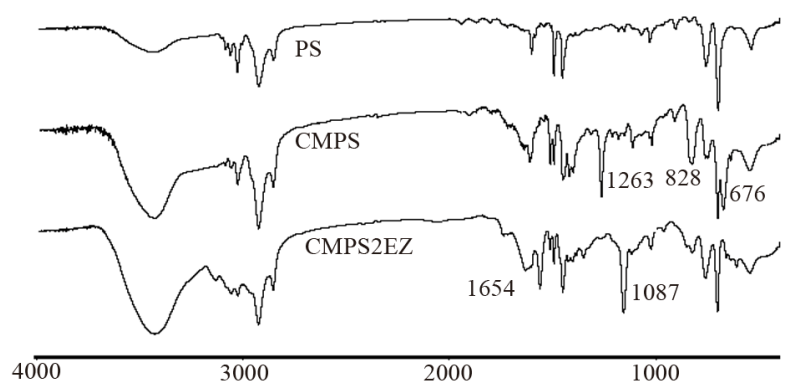

Figure 1. FTIR spectra of PS, CMPS, CMPS2EZ. 
of obtained CMPS resins were $3.72 \mathrm{mmol} / \mathrm{g}$.

\subsection{Weight Analysis of CMPS2EZ Resins}

Gravimetric analysis showed that after the reaction, the weight of CMPS resins increased from $4.064 \mathrm{~g}$ to $5.464 \mathrm{~g}$, which indicated that $99.46 \%$ of methyl chloride was reacted with $\mathrm{N}$-diethyl imidazole.

\subsection{Drawing Standard Curves}

Fresh solutions of iodine were prepared before each series of measurements by dissolving precisely $4.482 \mathrm{~g}$ of $\mathrm{KI}(0.027 \mathrm{~mol})$ and $1.7145 \mathrm{~g}$ of $\mathrm{I}_{2}(0.00675 \mathrm{~mol})$ in 250 $\mathrm{ml}$ volume of distilled water. All solutions were kept in the dark except during sampling. And the molar ratio of $\mathrm{KI}$ and $\mathrm{I}_{2}$ was $4: 1$ throughout this work. It is known that in aqueous solutions, the following equilibria can exist:

$$
\begin{gathered}
\mathrm{I}^{-}+\mathrm{I}_{2} \leftrightarrow \mathrm{I}_{3}^{-} \\
\mathrm{I}^{-}+2 \mathrm{I}_{2} \leftrightarrow \mathrm{I}_{5}^{-}
\end{gathered}
$$

In neutral and acidic solutions these species are stable, but at high $\mathrm{pH}$, iodine (as well as $\mathrm{I}_{3}^{-}$and $\mathrm{I}_{5}^{-}$) can disproportionate into iodide and $\mathrm{IO}_{x}^{-}$species, accordingly alkaline conditions was avoided in this paper.

The UV-vis absorption spectra of water samples were recorded on a Shimadzu 2550 spectrophotometer. Figure 2 shows a room temperature $\mathrm{UV}$-vis absorption spectrum for the $1 \mathrm{mmol} / \mathrm{L}$ of $\mathrm{I}_{3}^{-}$and $0.5 \mathrm{mmol} / \mathrm{L}$ of $\mathrm{I}_{2}$ solution. Three bands appeared in the UV-vis absorption spectrum. The band centered at $220 \mathrm{~nm}$ is attributed to $\mathrm{I}^{-}$, the bands at 288 and $351 \mathrm{~nm}$ are due to $\mathrm{I}_{3}^{-}$[10]. Accordingly, the amount of $\mathrm{I}_{3}^{-}$absorbed in the samples was monitored by recording UV-vis spectra of $\mathrm{I}_{3}^{-}$at $288 \mathrm{~nm}(2 \mathrm{ml}$ of $\mathrm{I}_{3}^{-}$solution diluted to $\left.25 \mathrm{ml}\right)$, and by mounting these into the inside wall of a quartz cell $(1 \mathrm{~cm} \times 1 \mathrm{~cm} \times 3 \mathrm{~cm})$. The results showed that the concentration of $\mathrm{I}_{3}^{-}$at 0.334 $\sim 1.112 \mathrm{mmol} / \mathrm{L}$ range of absorbance has good linear relationship, correlation coefficient $r=0.99954$.

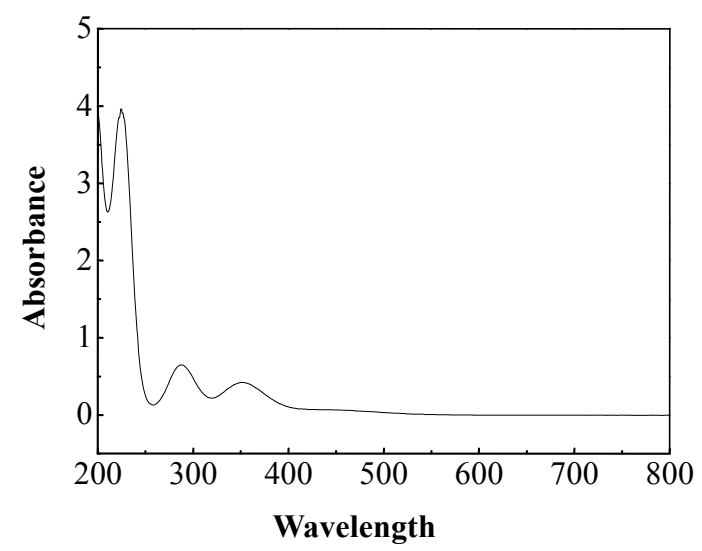

Figure 2. UV-vis absorption spectrum for the $1 \mathrm{mmol} / \mathrm{L}$ of $\mathrm{I}_{3}^{-}$and $0.5 \mathrm{mmol} / \mathrm{L}$ of $\mathrm{I}_{2}$ solution.

\subsection{Optimum pH of Iodine Uptake}

The adsorption amounts were calculated according to the following Equation (1)

$$
Q=\frac{\left(C_{0}-C\right) V}{W}
$$

where $Q$ is the adsorption amount $(\mathrm{mmol} / \mathrm{g}), C_{0}$ and $C$ the initial and concentrations of $\mathrm{I}_{3}^{-}$in solution when the contact time is $t$, respectively (mmol/L), $V$ the volume (L), $W$ the weight of resin beads $(\mathrm{g})$.

The effect of the acidity medium on the uptake of iodine on CMPS2EZ resins were studied at $20^{\circ} \mathrm{C}$. The obtained results are given in Figure 3. The uptake of iodine found to be higher at the natural water but lower uptake values were obtained at acidic medium, indicated that the higher uptake at the natural water may be attributed to the presence of $\mathrm{H}^{+}$distribution the coefficient towards solution directions, thus reducing the iodine adsorption capacity.

\subsection{Sorption Kinetics}

A study of adsorption kinetics is desirable as it provides information about the mechanism of adsorption, which is important for efficiency of the process. The adsorption kinetics of CMPS2EZ resins for iodine at different temperature was studied in this part.

Figure 4 shows that adsorption equilibriums of CMPS2EZ resins for iodine were established in $10 \mathrm{~h}$, while the saturation adsorption capacities didn't increase followed with the increasing of temperature as we expected. A possible explanation for this is that the diffusion rate of iodine is enhanced by increasing temperature, so iodine can approach and contact with the resins much faster at higher temperature. Consequently, the complexes quickly formed on the surface and outer layer of the resins at high temperature, which prevent the iodine diffusing into the inside of the resins, so the value of adsorption capacities at highest temperature, is not the largest.

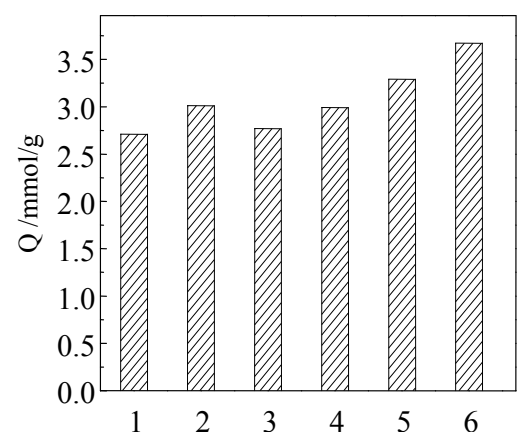

Test condition: (1) $5 \mathrm{mmol} / \mathrm{L} \mathrm{HCl},(2) 5 \mathrm{mmol} / \mathrm{L} \mathrm{H}_{2} \mathrm{SO}_{4}$, (3) $500 \mathrm{mmol} / \mathrm{L} \mathrm{CH}_{3} \mathrm{COOH}$, (4) $50 \mathrm{mmol} / \mathrm{L} \mathrm{CH}_{3} \mathrm{COOH}$, (5) $5 \mathrm{mmol} / \mathrm{L} \mathrm{CH}_{3} \mathrm{COOH},(6) \mathrm{H}_{2} \mathrm{O}$

Figure 3. The effect of the acidity medium on the uptake of CMPS2EZ resins for iodine. 
Analyzed data in Figure 5 by the procedure given by Helfferich [11] and Reichenberg [12]. The following Equations (2) were used:

$$
\begin{aligned}
F & =1-\frac{6}{\pi^{2}} \sum_{n=1}^{\infty} \frac{1}{n^{2}}\left[\frac{-D_{i} t \pi^{2} n^{2}}{r_{0}^{2}}\right] \\
& =1-\frac{6}{\pi^{2}} \sum_{n=1}^{\infty} \frac{1}{n^{2}} \exp \left(-n^{2} B_{t}\right)=\frac{Q_{t}}{Q_{0}}
\end{aligned}
$$

where $F$ is the fractional attainment of equilibrium at time $t$ and is obtained by the expression, $Q_{t}$ is the amount of adsorption taken up at time $t$ and $Q_{0}$ is the maximum equilibrium uptake and $D_{i}$ is the effective diffusion coefficient of iodine in the adsorbent phase; $r_{0}$ is the radius of the adsorbent particle, assumed to be spherical; and $n$ is an integer that defines the infinite series solution. $B_{t}$ values were obtained for each observed value of $F$ from Reichenberg's table and the results were plotted. The linearity test of $B_{t}$ vs. time plots was employed to distinguish between the film diffusion and particle diffusion controlled adsorption [8]. If the plot of $B_{t}$ vs. time is a straight line passing through the origin, then the adsorption rate is governed by particle diffusion mechanism, otherwise it is governed by film diffusion. From Figure $\mathbf{6}$ it could be seen that the four straight lines under different temperatures did not pass through the origin, indicating the rate controlling step not to be particle diffusion, but film diffusion. The linear equations and correlation coefficients $R_{2}$ were listed in Table 1 .

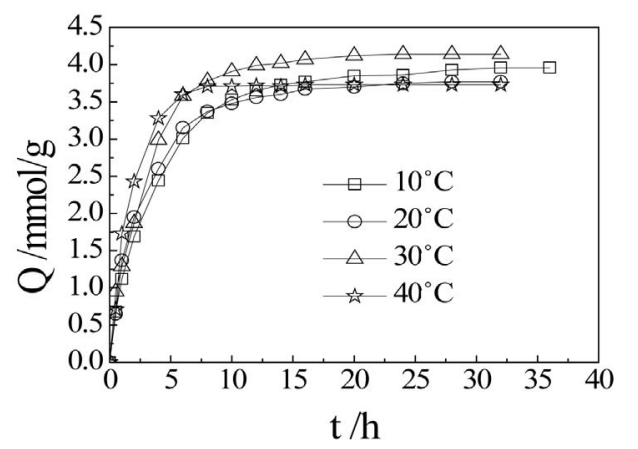

Figure 4. The sorption kinetics of CMPS2EZ for iodine.

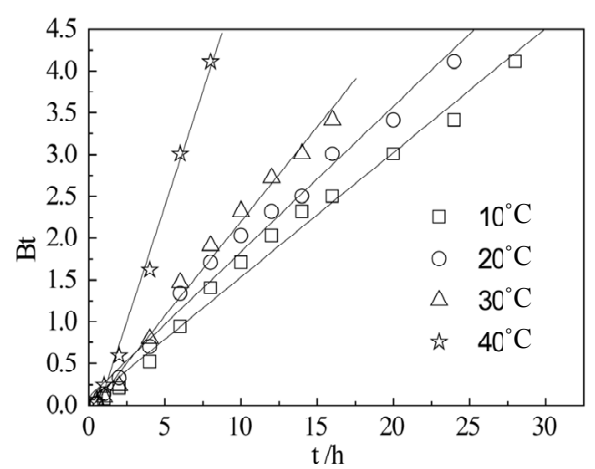

Figure 5. The Bt-t of CMPS2EZ for iodine.
Adsorption kinetics can be modeled by several models, including pseudo first-order rate equation and pseudo second-order rate equation given below as Equations (3) and (4), respectively.

$$
\begin{gathered}
\log \left(Q_{0}-Q\right)=\log Q_{0}-\frac{K}{2.303} t \\
\frac{t}{Q}=\frac{1}{K_{2} Q_{0}{ }^{2}}-\frac{1}{Q_{0}} t
\end{gathered}
$$

where $k_{1}$ is the rate constant of pseudo first-order adsorption $\left(\mathrm{h}^{-1}\right), K_{2}$ the rate constant of pseudo second-order adsorption $(\mathrm{g} / \mathrm{mmol}), Q_{0}$ and $Q$ are the adsorption amount at equilibrium and at time $t$, respectively. Both the models were used to fit the kinetics curves and the results showed that both the models were suitable since the values of $R_{2}$ could be regarded as a measure of the goodness-of-fit of experimental data on the kinetic models. The straight-lines of pseudo second-order kinetic model are showed in Figures 6 and 7. The parameters calculated according to the model are listed in Table 2.

Table 1. The Bt-t of CMPS2EZ for iodine.

\begin{tabular}{cccc}
\hline Temperature $/{ }^{\circ} \mathrm{C}$ & Linear equation & $R_{2}$ & Intercept error \\
\hline 10 & $B_{t}=0.1488 t-0.0476$ & 0.9884 & 0.0686 \\
20 & $B_{t}=0.1738 t-0.1012$ & 0.9858 & 0.0805 \\
30 & $B_{t}=0.2262 t-0.0601$ & 0.9905 & 0.0709 \\
40 & $B_{t}=0.5524 t-0.3765$ & 0.9930 & 0.1042 \\
\hline
\end{tabular}

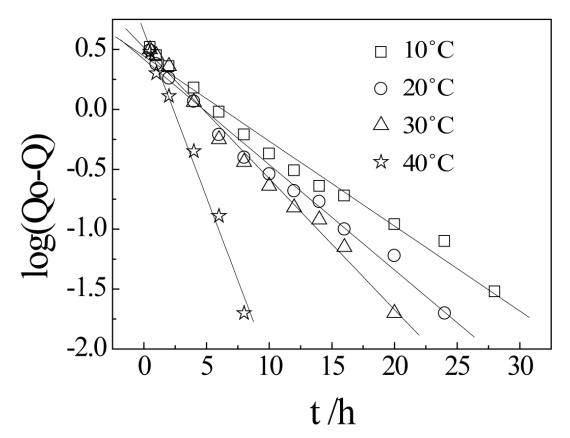

Figure 6. The pseudo first-order kinetic models of CMPS2EZ for iodine.

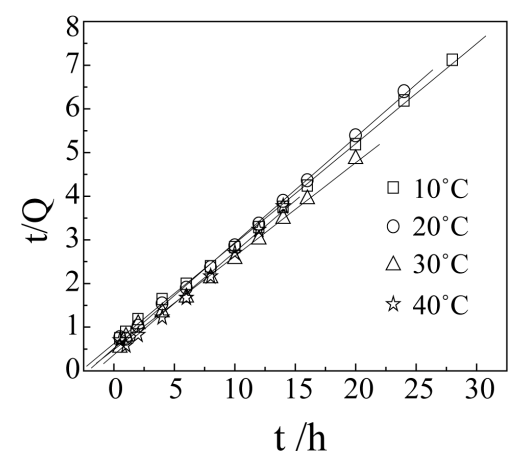

Figure 7. The pseudo second-order kinetic models of CMPS2EZ for iodine. 


\subsection{Adsorption Thermodynamics}

It can be seen from Figure 8 that temperature had a significant effect on the adsorption capacities, that is, the adsorption capacities increased with increasing temperature. But when the temperature is higher than $30^{\circ} \mathrm{C}$, adsorption capacities didn't increase with the increasing of the temperature.

As we know, the Langmuir Equation (5) is applicable to homogeneous adsorption where the adsorption of each sorbate molecule onto the surface had equal adsorption activation energy. While the Freundlich Equation (6) is employed to describe heterogeneous systems and reversible adsorption, which is not restricted to the formation of monolayer. The parameters for the two isotherms obtained from Figures 9 and $\mathbf{1 0}$ are presented in Table 3.

Table 2. Parameters of pseudo first-order and pseudo second-order models of CMPS2EZ for iodine.

\begin{tabular}{cccc}
\hline \multirow{2}{*}{ Temperature $/{ }^{\circ} \mathrm{C}$} & \multicolumn{3}{c}{ Pseudo first-order model } \\
\cline { 2 - 4 } & $k 1$ & $Q_{0}$ & $R_{2}$ \\
\hline 10 & 0.1634 & 2.78 & 0.9643 \\
20 & 0.2025 & 2.62 & 0.9744 \\
30 & 0.2519 & 3.28 & 0.9815 \\
40 & 0.6373 & 4.47 & 0.9706 \\
\hline \multirow{2}{*}{ Temperature $/{ }^{\circ} \mathrm{C}$} & \multicolumn{3}{c}{ Pseudo second-order model } \\
\cline { 2 - 4 } 10 & 0.0840 & $Q_{0}$ & $R_{2}$ \\
20 & 0.1133 & 4.37 & 0.9976 \\
30 & 0.0944 & 4.67 & 0.9974 \\
40 & 0.1504 & 4.25 & 0.9936 \\
& \multicolumn{3}{c}{0.9837} \\
\hline
\end{tabular}

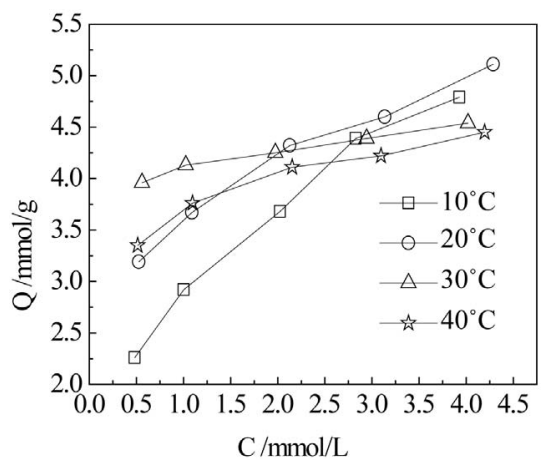

Figure 8. Isothermal adsorption of CMPS2EZ for iodine.

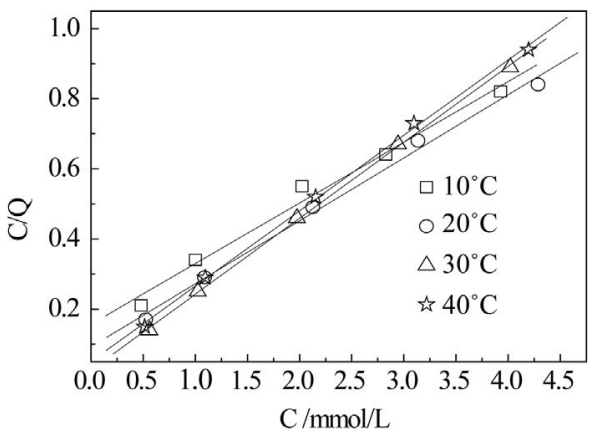

Figure 9. The Langmuir isotherms of CMPS2EZ for iodine.

$$
\begin{gathered}
\frac{C}{Q_{t}}=\frac{1}{b Q_{0}}+\frac{C}{Q_{0}} \\
\ln Q_{t}=\ln K+\frac{1}{n} \ln C
\end{gathered}
$$

where $Q$ is the adsorption capacity $(\mathrm{mmol} / \mathrm{g}), C$ the equilibrium concentration of $\mathrm{I}_{3}^{-}(\mathrm{mmol} / \mathrm{L}), Q_{0}$ the saturated adsorption capacity $(\mathrm{mmol} / \mathrm{g}), b$ an empirical parameter, $n$ the Freundlich constant, and $K$ the binding energy constant reflecting the affinity of the resins to iodine. It can be seen that in the case of CMPS2EZ resins for iodine, both Freundlich equations and Langmuir equations could well interpret the adsorption of CMPS2EZ for iodine.

\section{Conclusion}

A new N-diethyl imidazole modified PS resins was synthesized in this paper. The composition, structure, and properties of the resulting resins were studied by means of Fourier transform infrared (FTIR) and elemental analysis methods. The results showed that the resins hold the adsorption function group of quaternary ammonium structure. Meanwhile, the adsorption properties of the resulting resins for iodine were investigated. The results showed that the uptake of iodine found to be higher at the

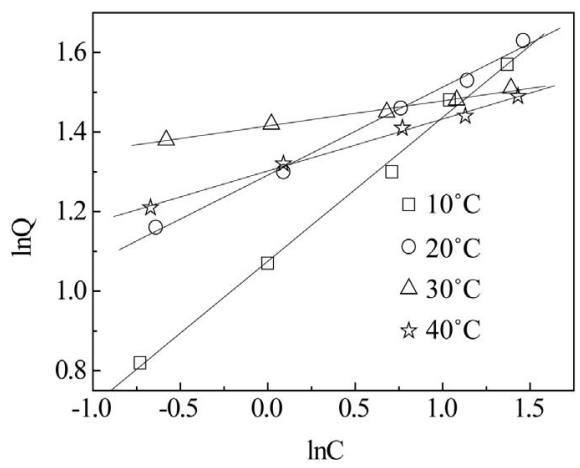

Figure 10. The Freundlich isotherms of CMPS2EZ for iodine.

Table 3. Langmuir and Freundich parameters of CMPS2EZ for iodine.

\begin{tabular}{cccc}
\hline \multirow{2}{*}{ Temperature $/{ }^{\circ} \mathrm{C}$} & \multicolumn{3}{c}{ Langmuir } \\
\cline { 2 - 4 } & $Q_{0}$ & $b$ & $R_{2}$ \\
\hline 10 & 5.78 & 1.1054 & 0.9865 \\
20 & 5.56 & 1.9419 & 0.9946 \\
30 & 4.62 & 8.3395 & 0.9995 \\
40 & 4.65 & 4.2681 & 0.9987 \\
\hline \multirow{2}{*}{ Temperature $/{ }^{\circ} \mathrm{C}$} & & Freundlich & \\
\cline { 2 - 4 } 10 & & $1 / n$ & $R_{2}$ \\
\hline 20 & 2.9304 & 0.3616 & 0.9948 \\
30 & 3.6393 & 0.2210 & 0.9953 \\
40 & 4.1181 & 0.0630 & 0.9862 \\
& 3.6774 & 0.1305 & 0.9958 \\
\hline
\end{tabular}


natural water than at acidic medium; the adsorption capacities increased with the increasing of temperature, but when the temperature is higher than $30^{\circ} \mathrm{C}$, adsorption capacities didn't increase; the adsorption kinetics of the resins can be modeled by pseudo first-order rate equation and pseudo second-order rate equation wonderfully; Both Freundlich equations and Langmuir equations could well interpret the adsorption of resins for iodine.

\section{REFERENCES}

[1] K. V. Ellis and A. P. Cotton, "Iodine Disinfection of Poor Quality Waters," Water Research, Vol. 27, No. 3, 1993, pp. 369-375. doi:10.1016/0043-1354(93)90036-H

[2] A. Sengupta and M. Holtz, "Temperature-Dependent Resonance Raman Study of Iodine-Doped Poly(Vinyl Alcohol) Films," Chemical Physics Letters, Vol. 263, No. 1-2, 1996, pp. 25-32. doi:10.1016/S0009-2614(96)01187-6

[3] J. D. Song and R. Ryoo, "Anion Binding Properties of Poly(Vinyl Pyrrolidone) in Aqueous Solution Studied by Halide NMR Spectroscopy," Macromolecules, Vol. 24, No. 8, 1991, pp. 1727-1730. doi:10.1021/ma00008a006

[4] D. L. A. Faria and H. A. C. Gil, "The Interaction between Polyvinylpyrrolidone) and $\mathrm{I}_{2}$ as Probed by Raman Spectroscopy," Journal of Molecular Structure, Vol. 478, No. 1-3, 1999, pp. 93-98. doi:10.1016/S0022-2860(98)00755-8

[5] H. Sakai and T. Matsuyama, "An 129I Mossbauer Spectroscopic Study of Iodine Doped in Poly(Vinylpyridines)," The Journal of Chemical Physics, Vol. 75, No. 10, 1981, pp. 5155-5159. doi:10.1063/1.441864
[6] S. Palaniappan and D. N. Sathyanarayana, "Polymeric Charge Transfer Complexes: A Study by Electronic Absorption Spectroscopy," Polymer, Vol. 31, No. 8, 1990 , pp. 1401-1405. doi:10.1016/0032-3861(90)90140-T

[7] H. M. Tsai and H. W. Ko, "Studies on the Electrical Properties of PVP-I2 Complexes," Journal of Power Sources, Vol. 26, No. 3-4, 1989, pp. 557-561. doi:10.1016/0378-7753(89)80178-8

[8] N. Kawabata, "Capture of Micro-Organisms and Viruses by Pyridinium-Type Polymers and Application to Biotechnology and Water Purification," Progress in Polymer Science, Vol. 17, No. 1, 1992, pp. 1-34. doi:10.1016/0079-6700(92)90015-Q

[9] G. J. Li and J. R. Shen, "Study of Pyridinium-Type Functional Polymers IV: Behavioral Features of the Antibacterial Activity of Insoluble Pyridinium-Type Plymers," Journal of Applied Polymer Science, Vol. 78, No. 3, 2000, pp. 676-684. doi:10.1002/1097-4628(20001017)78:3<676::AID-APP2 40>3.0.CO;2-E

[10] S. Archita, H. Mark and L. Edward, "Chemical Physic Temperature-Dependent Resonance Raman Study of Iodine-Doped Poly(Vinyl Alcohol) Films," S Letters, Vol. 263, No. 1-2, 1996, pp. 25-32.

[11] F. Helfferich, "Ion-Exchange," McGraw-Hill, New York, 1962.

[12] D. Reichenberg, "Properties of Ion-Exchange Resins in Relation to Their Structure III Kinetics of Exchange," Journal of the American Chemical Society, Vol. 75, No. 3, 1953, pp. 589-597. doi:10.1021/ja01099a022 